\title{
Norois
}

Environnement, aménagement, société

$231 \mid 2014$

Les modes d'habiter à l'épreuve de la durabilité

\section{Habiter l'espace touristique et porter attention à la ressource en eau potable. Analyse qualitative des positions des touristes dans le Morbihan (France)}

Inhabiting a tourist space and caring for drinking water resources. Qualitative analysis of tourist positions in Morbihan area (France)

\section{Xavier Michel}

\section{OpenEdition}

Journals

\section{Édition électronique}

URL : http://journals.openedition.org/norois/5082

DOI : $10.4000 /$ norois.5082

ISBN : 978-2-7535-3599-2

ISSN : $1760-8546$

Éditeur

Presses universitaires de Rennes

Édition imprimée

Date de publication : 30 septembre 2014

Pagination : 45-60

ISBN : 978-2-7535-3465-0

ISSN : 0029-182X

Référence électronique

Xavier Michel, « Habiter l'espace touristique et porter attention à la ressource en eau potable. Analyse qualitative des positions des touristes dans le Morbihan (France) », Norois [En ligne], 231 | 2014, mis en ligne le 30 juin 2016, consulté le 21 avril 2019. URL : http://journals.openedition.org/norois/5082 ; DOI : $10.4000 /$ norois. 5082 


\title{
Habiter l'espace touristique et porter attention à la ressource en eau potable Analyse qualitative des positions des touristes dans le Morbihan (France)
}

\author{
Inhabiting a Tourist Space and Caring for Drinking Water Resources \\ Qualitative Analysis of Tourist Positions in Morbihan Area (France)
}

\author{
Xavier MicheL
}

ESO, UMR 6590 CNRS, équipe ESO-Caen, Normandie Université (UNICAEN), Maison de la Recherche en

Sciences Humaines - Esplanade de la Paix, Campus 1-14032 Caen Cedex, France (xavier.michel@unicaen.fr)

Résumé : Cet article met en relation les modes d'habiter touristique avec le rapport à la ressource en eau développé par les touristes séjournant dans des destinations littorales du département du Morbihan (France). À partir d'entretiens qualitatifs, l'objectif de la recherche est de montrer que les modes d'habiter la destination touristique sont à comprendre en examinant le parcours de mobilité de chaque individu. Les mobilités ont des effets sur la relation à l'environnement : entre attachement et détachement, entre préoccupation pour la nature, la gestion technique et/ou les autres individus dans l'espace social.

Abstract: This article explores how tourists inhabit coastal places of the Morbihan area (France) and their environmental connections with water resources. Based on in-depth interviews with tourists, we want to demonstrate that the ways tourists inhabit places can be understood through the analysis of their mobility. Mobility impacts the relationship to the environment: depending on the attachment and the detachment to places, the concern for nature, technical management and/or other individuals in social space.

Mots clés : Habiter, tourisme, mobilité, environnement, ressource, eau, monde.

Keywords: Inhabiting, tourism, mobility, environment, resource, water, world.

\section{INTRODUCTION}

Dans une société où la préoccupation pour l'environnement est affichée, comment les touristes, qui, pour certains, ne connaissent pas bien l'environnement dans lequel ils séjournent, peuvent porter une attention à une ressource environnementale? La question a été posée au sujet de la ressource en eau potable, dans le cadre d'un programme de recherche ${ }^{1}$ réalisé dans le Morbihan. Comme d'autres destinations touristiques, la partie littorale de ce département connaît une fréquentation esti-

\footnotetext{
1. Cette recherche, intitulée «Aléa sécheresse et risque de pénurie d'eau dans un territoire touristique : le Morbihan », a été menée dans le cadre du programme Risque, décision, territoire n² 2 , entre 2008 et 2010.
} 
vale importante ${ }^{2}$ (figure 1). Dans les destinations touristiques, se pose l'enjeu de la consommation de l'eau potable dans un contexte de changement climatique (Gössling, 2006; Gössling et al., 2012). Si l'alimentation en eau potable est assurée par un réseau d'interconnexions (figure 1) dans la majorité du département du Morbihan, le risque de pénurie d'eau potable a été mis en évidence pour les territoires insulaires, à partir de travaux de recherches (Chiron, 2007a et b) et d'analyses des acteurs locaux. Le principal événement fut la pénurie d'eau (entendue au sens d'un manque relatif d'eau potable) à Belle-île au second semestre 2005 et au premier semestre 2006, à la suite de la sécheresse de 2005 (Chiron, 2007a).

Les usagers ne savent pas tous quelle est l'organisation de d'alimentation en eau potable. Il existe trois leviers pour organiser et sécuriser cette alimentation dans le territoire du Morbihan :

- le développement des interconnexions à échelle départementale et régionale (figure 1),

- le renouveau des dispositifs de pompage et de captage locaux, afin de réduire la dépendance aux approvisionnements distants,

- les économies d'eau des usagers, par les équipements hydro-économes et par l'attention portée à la consommation au robinet.

Cette recherche se situe dans le cadre de ce troisième levier. L'enjeu est d'identifier et d'expliquer comment les populations touristiques pensent à et parlent de la ressource en eau potable. Pensent-elles à l'usage parcimonieux et au partage de cette ressource environnementale ou pas? Les publics touristiques manqueraient de conscience environnementale et n'auraient pas assez conscience des effets de leurs pratiques sur l'environnement (Miller et al., 2010). Il ne s'agit pas ici de considérer les touristes comme étant a priori moins attentionnés, ayant des usages dispendieux en eau, par contraste avec la population permanente. L'objectif est de travailler l'effet de leur statut d'usager temporaire du Morbihan sur leur rapport à la ressource en eau potable, sachant qu'ils engendrent, par l'addition de population, une demande élevée sur la bande littorale en été.

2. Le Morbihan arrive, au moment du pic de fréquentation touristique estivale, dans le groupe des départements recevant le plus de touristes (entre 500000 et 800000 touristes), soit au 6 rang national (après le Var, l'Hérault, la Charente-Maritime, la Vendée et les Pyrénées-Orientales) : données départementales mensuelles, et carte du solde touristique au 15 août 2005, [http://www.christophe-terrier.com/presence/].
Les statuts des usagers temporaires du littoral sont variés : entre le touriste occasionnel et le résident secondaire régulier, remettant en question l'existence d'une seule population touristique (Vacher et Vye, 2012). La recherche conduite ici s'est concentrée sur les résidents secondaires et les touristes séjournant en camping. La figure 1 traduit la répartition spatiale de ces catégories dans le Morbihan, chaque cercle, par commune, représentant le total de la capacité d'accueil en nombre de résidences secondaires et d'emplacements de campings ${ }^{3}$. Certains individus ont en mémoire des épisodes de sécheresse passés (renvoyant au phénomène climatique), et d'expériences de pénurie d'eau (renvoyant au phénomène social), dans le Morbihan ou ailleurs. Nous faisons l'hypothèse que les différentes sphères territoriales des individus agissent sur la manière de considérer la ressource en un lieu précis.

L'espace touristique est compris, dans cet article, non seulement comme la destination « Morbihan ", mais aussi comme une agrégation des différents espaces vécus des individus touristes, fréquentés par le passé, et parfois imaginés sans avoir été fréquentés. Ces espaces vécus ne sont pas définis a priori; l'objectif de la recherche est de les identifier. À partir de ce cadre de réflexion, quelle attention à la ressource en eau potable peut-on attendre de la part de populations pratiquant temporairement une destination touristique?

\section{Construire un CADre DE RECHERCHE ARTICULANT L'HABITER TOURISTIQUE ET LA RESSOURCE EN EAU}

\section{Des destinations morbihannaises entre aspects génériques du tourisme littoral et spécificités locales}

Les littoraux du Morbihan font partie des destinations littorales estivales avec un seul pic principal de

\footnotetext{
3. Source : INSEE. Outres les résidences secondaires et les emplacements dans les campings, les statistiques comprennent aussi le nombre de chambres d'hôtel (mais pas les autres formes d'hébergement touristique), non repris ici pour l'élaboration de la figure 1, car les publics hébergés en hôtel n'ont pas été interrogés lors de l'enquête. Cela ne modifie pas la répartition géographique d'ensemble de la présence touristique dans le Morbihan en été, car on observe dans tous les cas une concentration importante sur la bande littorale.
} 


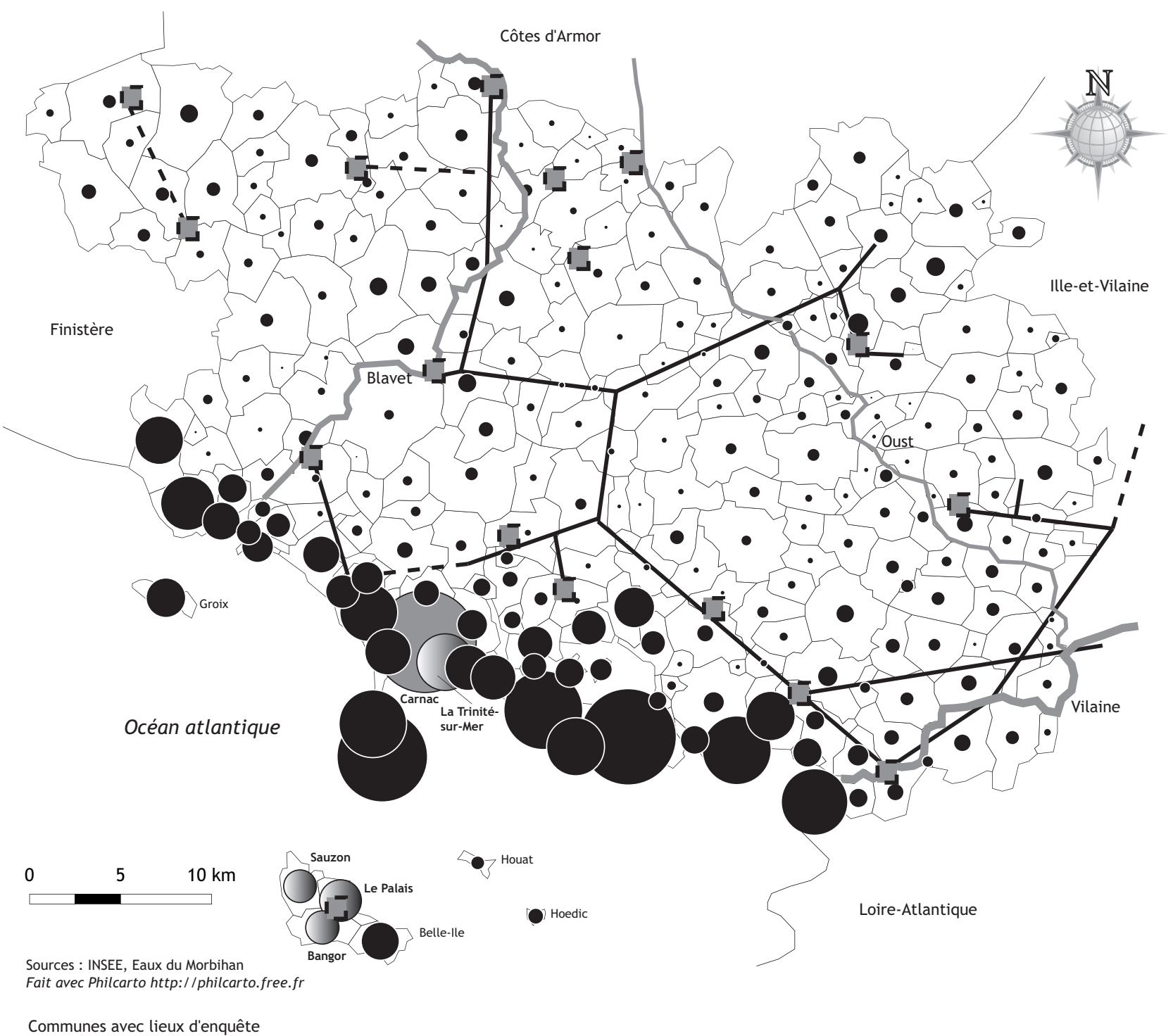

Communes avec lieux d'enquête

sauzon

Nombre de résidences secondaires et emplacements de camping en 2009

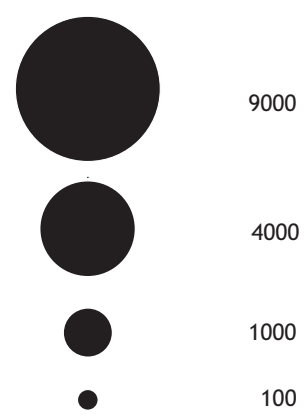

Feeder d'eau potable en 2013

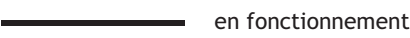

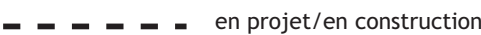

Usine de production d'eau potable en 2013

ב

Principaux cours d'eau

Figure 1 : Tourisme et alimentation en eau potable dans le Morbihan Tourism and drinking water supply in Morbihan area 
fréquentation touristique en été (Terrier, 2006) ${ }^{4}$ et un espace d'émission provenant à la fois de la région parisienne et des régions proches, notamment des grandes villes de Rennes et Nantes (Potier, 2002). L'équipement d'hébergement est monté en gamme, pour répondre à une demande de confort supplémentaire par rapport à l'hébergement de plein air. Ce processus est socialement discriminant, accessible pour les « classes » à mobilité choisie (Ollivro, 2005). Comme d'autres destinations littorales, le Morbihan est aussi marqué par un fort contraste de fréquentation entre la côte et l'intérieur (Billaud et Michel, 2010). En termes d'usage de l'eau potable, il est socialement et économiquement marqué par la mise en œuvre d'une transition écologique ${ }^{5}$, avec les équipements hydro-économes et la sensibilisation des habitants aux pratiques d'économie dans l'usage de l'eau potable ${ }^{6}$. Dans le secteur touristique du Morbihan, cette sensibilisation a été observée et analysée par une enquête auprès des hébergeurs professionnels ${ }^{7}$.

D'autre part, les données hydrologiques et hydrographiques du département sont spécifiques. Le Morbihan relève d'un régime pluvial océanique avec une forte variabilité interannuelle de la ressource (Hellier et al., 2009). La disponibilité de cette dernière est définie par rapport à des bassins-versants ayant, au sein d'une péninsule, une faible superficie. La situation a été améliorée, à l'échelle de la majorité du département, par une interconnexion du réseau d'alimentation, bénéficiant notamment de l'apport du volume d'eau de la Vilaine à partir de la station de Férel, ainsi que d'un apport du fleuve Blavet (Figure 1). L'origine de ce réseau n'est pas due à une pénurie avérée, mais, selon le Syndicat départemental de l'eau, à une volonté de « sécurisation et de mutualisation des approvisionnements à

4. Christophe Terrier évalue la population présente maximale le 3 août 2005 dans le Morbihan à 1,1 million de personnes présentes, par rapport à une base de résidents permanents de 677500 habitants, soit $+63,8 \%$.

5. « La transition écologique implique un changement du modèle économique actuel vers une économie à la fois plus compétitive et respectueuse de l'environnement, permettant une croissance durable et une amélioration quantitative et qualitative de l'emploi ». [http://www.developpementdurable.gouv.fr/Emploi-et-transition-ecologique.html].

6. Le Morbihan s'inscrit ici dans une démarche régionale, où des villespilotes d'économie d'eau ont été définies. Parmi les villes bretonnes choisies, figurent Lorient, Vannes et Pontivy : Agence de l'eau Loire-Bretagne et Région Bretagne (1999). Economiser l'eau dans la ville et l'habitat Guide méthodologique, [http://www.eau-loire-bretagne.fr/espace_documentaire/ documents_en_ligne/guides_economies_deau/economie_eau_habitat.pdf].

7. Enquête réalisée dans le cadre de la recherche «Aléa sécheresse et risque de pénurie d'eau dans un territoire touristique : le Morbihan » (Billaud, Michel, 2010) : sur 135 hébergeurs répondants, 58\% d'entre eux ont déclaré mener une action de sensibilisation à la préservation de l'eau potable auprès de leur clientèle. l'échelle départementale » ${ }^{8}$, dans un contexte général de croissance urbaine et touristique. Le dispositif inclut néanmoins aussi l'objectif de pallier les déficits pluviométriques exceptionnels.

La variabilité interannuelle de la ressource conduit à l'éventualité de sécheresses dans la France océanique, qui est démontrée notamment à partir du critère de valeur moyenne de déficience d'évaporation : la côte sud de la Bretagne est parmi les premières régions exposées (Dubreuil, 1997). À ceci s'ajoute le facteur géologique : les zones schisteuses de l'est du département ne sont pas favorables à la constitution de réserves d'eau profondes (Mounier et al., 1993). Ces contraintes ont contribué au développement d'un réseau d'alimentation interconnecté. Ce réseau a permis d'écarter les manifestations d'une pénurie d'eau dans l'espace continental, même si les manifestations de la sécheresse donnent lieu à des mesures de restrictions et sont perçues par les populations ${ }^{9}$.

Dans les îles qui ne bénéficient pas de l'alimentation en eau potable depuis le continent, l'importance de la population saisonnière a conduit à un risque de pénurie d'eau (Chiron, 2007a et 2007b). Cependant le risque a déjà été réduit, d'un point de vue technique, sur les plus grandes îles (Belle-Île notamment), par la constitution de retenues d'eau douce ${ }^{10}$ et, plus récemment, par la réactivation de stations de pompage de fond de vallons, même si les perspectives de hausse de la population présente amènent toujours les élus et les gestionnaires du réseau d'eau potable à se poser la question de l'alimentation en eau potable et à prévoir des améliorations pour consolider le réseau existant.

\section{Que signifie habiter un espace touristique?}

Que savent les touristes de ces informations? Comment appliquer de bonnes pratiques en

8. «L'alimentation en eau potable », note du Conseil général du Morbihan, [http://www.morbihan.fr/dossier/default.aspx?idDos=51 1 id =239].

9. Les « grandes » sécheresses, des années 1976, 1989, 1995, 2005, sont dans les mémoires de certaines personnes (surtout celle de 1976). Mais, au cours de la dernière décennie, il faut citer aussi les différentes années où des arrêtés de restriction de l'eau potable ont été pris sur au moins une partie du territoire du Morbihan : 2003, 2005, 2006, 2010.

10. À Belle-Île-en-Mer, les trois retenues d'eau ont été formées par la construction de trois barrages : Bordilla en 1941, Antoureau en 1969, à la suite de l'essor du réseau d'alimentation et du tourisme, et Borfloc'h en 1993. Depuis 2010, un projet d'unité de dessalement est périodiquement évoqué pour renforcer les capacités d'alimentation en eau potable à Belle-Île (Ouest France, 2013) : [http://www.ouest-france.fr/Belle-Ille-unprojet-dusine-de-dessalement-nouveau-evoque-408266]. 
termes de durabilité des ressources, si l'espace pratiqué n'est pas bien connu? Pourquoi et comment faire comprendre l'enjeu d'un usage parcimonieux de la ressource dans un territoire où celle-ci est assez abondante hors des épisodes de sécheresse? Comment cohabiter avec les autres si la connaissance et les expériences des espaces diffèrent ${ }^{11}$ ?

L'habiter a été posé dans un sens plus diversifié que le seul habitat permanent, en englobant les déplacements, les pratiques éphémères des espaces, les séjours temporaires dans différents lieux. En travaillant sur l'habiter, les chercheurs analysent la construction d'une identité polyspatiale. Cette dimension avait déjà été mise en évidence à partir des années 1980 : «Nous utilisons des espaces multiples, et notre vie personnelle est la somme de ces espaces partiels [...]. Les moments des déplacements sont les vecteurs unifiants de l'ensemble » (Viard, 1984) ${ }^{12}$. Plus récemment, cette idée a été reprise, en parlant d'individualisme géographique et, à partir de la notion de lieu, en qualifiant l'habiter contemporain de polytopique (Stock, 2006). Le cadre d'analyse récemment proposé par Mathis Stock (2012) pour confronter le phénomène touristique à l'habiter nous paraît intéressant et opératoire. Le mode d'habiter s'exprime dans chaque espace de déplacement et placement : la destination touristique, au sens générique, est un des lieux où s'exercent les modes d'habiter. Le style d'habiter rassemble l'ensemble du trajet de mobilités d'un individu : la notion de «style d'habiter » fonctionne ainsi avec les différents espaces pratiqués, voire imaginés par chaque individu, avec ses différents « ici » et « ailleurs ». Le régime d'habiter exprime, lui, davantage les différentes normes régissant l'organisation sociale et spatiale d'une société. Dans cette recherche, nous nous concentrerons sur le mode et le style d'habiter et sur les liens qui peuvent être décelés entre ces deux notions. Les individus touristes rencontrés dans les destinations du Morbihan y développent ainsi des modes d'habiter, qui sont à la fois une partie de leur style d'habiter et

11. La notion de cohabitation est ici avancée au sens des relations entre tourisme et milieu d'accueil, non à partir de problèmes particuliers sur ce territoire. C'est l'enquête auprès des touristes qui a pour but d'identifier comment se traduit cette cohabitation.

12. Le dernier chapitre de l'ouvrage, intitulé : «L'individu polyspatial », s'appuie sur les notions d'espaces éclatés, multiples, partiels. Pour chaque individu, différents parcours forment un «territoire privé », dans une « vie polyspatiale ». en même temps influencés par ce style. L'enjeu de la recherche est de savoir comment.

Ce mode d'habiter est marqué, soit par un engagement et un attachement avec l'espace par la relation paysagère, comme le montre notamment Helen Maulion (2009) au sujet des résidents qui s'installent à Belle-île-en-Mer, soit par un détachement, une distanciation vis-à-vis de l'espace (Stock, 2005). Ces modes d'habiter sont à concevoir selon les préoccupations de chaque individu par rapport à des espaces qui peuvent être lointains physiquement tout en étant très présents dans l'esprit des individus pour établir une relation au monde par un assemblage d'espaces spécifique à chacun (Hoyaux, 2003). L'habiter touristique des individus n'est pas un système prenant seulement en compte les espaces touristiques; une interaction s'opère aussi avec les espaces du quotidien : le domicile actuel et les domiciles passés (Michel, 2005).

\section{Pourquoi mobiliser les modes et les styles d'habiter touristiques pour identifier les rapports à la ressource en eau?}

Les modes d'habiter opèrent des rapports à un environnement physique et social. « L'environnement a des dimensions variables, qui vont du globe au voisinage » (Emélianoff, 2013). La pratique d'un lieu met chacun en contact avec une matérialité de l'environnement qui peut être perçue sans limites internes, comme l'atmosphère. L'individu peut déployer une conscience environnementale, un être-là avec des éléments de la nature (l'atmosphère, la mer) représentés à partir du lieu ici, sans discontinuité jusqu'à l'étendue mondiale. Il importe de cerner « ce par rapport à quoi l'acteur situe son action » (Raymond, 2004), au niveau de la destination touristique expérimentée dans le Morbihan et au niveau des autres espaces. L'eau est considérée dans une approche plurielle, qui intègre les différentes imaginations matérielles appréhendées par Bachelard (1942) : eaux claires, profondes, mouvantes, mais aussi eaux composées avec un autre élément de la nature.

On a tendance à considérer que seuls les « écotouristes » formeraient un public ayant une faible empreinte écologique. L'enjeu est de dépasser cette opinion pour identifier un champ plus large d'«Environmental Friendly Tourists » (Dolnicar 
et al., 2008). Il s'agit enfin de se questionner sur l'influence des informations sur les changements environnementaux en termes de représentations et de pratiques des touristes (Buzinde et al., 2010). Au-delà de leurs mobilités, les individus sont aussi environnés d'informations et de natures mobiles (Urry, 2005), qu'ils reprennent dans leurs représentations. Les modes et les styles d'habiter sont ainsi à analyser de manière articulée pour évaluer les rapports que les touristes entretiennent avec la ressource en eau potable dans une destination touristique.

\section{Comment évaluer les modes d'habiter touristiques en relation avec la question de l'eau?}

Afin d'identifier les relations entre l'habiter touristique et la ressource en eau, 52 entretiens semidirectifs ont été menés en juillet et août 2009 auprès de touristes, dans trois destinations du Morbihan : 25 à Belle-île-en-Mer (répartis entre Le Palais, Sauzon et Bangor), 21 à La Trinité-sur-Mer et 6 à Carnac. La majorité des personnes (38) a été interrogée dans les campings de ces localités, 14 l'ont été dans les résidences secondaires ou prêtées par des proches. L'évaluation de ces modes d'habiter a pour but de comprendre les différentes relations développées avec l'environnement et la ressource en eau potable; non de les mesurer. Les questionnements développés dans les entretiens portaient sur les représentations et les pratiques de l'environnement, et notamment de la ressource en eau potable. Ils s'attachaient aussi à saisir l'opinion des individus au sujet des acteurs les mieux à même d'agir pour la préservation et la durabilité de cette ressource.

A travers les discours développés, les individus font part de leurs lieux de résidence principale et de leurs espaces de mobilité touristique. Ils évoquent les souvenirs d'événements passés liés à l'environnement (épisodes de sécheresse). L'objectif est de partir des questions d'environnements pour faire émerger ce qui compte en matière d'expériences et d'imaginaires chez chaque individu. La temporalité quotidienne, comme les moments exceptionnels, structurent les rapports aux environnements et, plus spécialement, à la ressource en eau potable. Vivre dans telle région et avoir tel parcours d'expériences touristiques amène chacun à développer des représentations de l'environnement et de cette ressource.

Les rencontres avec les individus ont été prévues dans un objectif qualitatif, qui ne répondait pas aux exigences d'un échantillonnage représentatif de la population touristique du Morbihan. Le choix de deux types de destinations contrastées, un espace littoral urbanisé sur le continent et un espace insulaire, a été guidé par la volonté d'analyser les différences d'équipements, de fréquentation touristique, mais aussi de mettre en évidence des points communs entre ces destinations. L'enjeu de la recherche est ainsi de faire apparaître plusieurs types d'individus ayant des modes d'habiter touristiques et des rapports à la ressource en eau potable semblables ${ }^{13}$.

Les entretiens ont été effectués sur place, dans les lieux d'hébergement touristique, en face-à-face et enregistrés sur support sonore. La durée des entretiens varie entre 40 minutes et $1 \mathrm{~h} 20$. Le lieu est significatif du mode d'habiter touristique quand il s'agit de résidents secondaires ayant acquis leur propriété depuis quelques années, ou bien, à l'inverse, quand il s'agit de touristes de passage étant dans une pratique de découverte sans attachement à la destination. Il est à relativiser quand il est différent de celui utilisé d'habitude et constitue alors un écart par rapport à l'expérience touristique habituelle dans le Morbihan. Le déroulement des entretiens s'est fait en tenant compte aussi de la présence de plusieurs personnes (famille, groupe d'amis) souhaitant répondre à l'enquête. Un répondant principal a alors été désigné au début de l'entretien. Les autres membres du groupe n'étaient pas interdits de parole, à partir du moment où le répondant principal jouait son rôle, et pouvaient même apporter des informations factuelles intéressantes pour compléter les réponses sur les pratiques ${ }^{14}$.

Les entretiens ont été réalisés au cours d'un été sans chaleurs importantes, avec des passages de temps couvert, et avec parfois des averses importantes. À Belle-Île-en-Mer, la question de l'usine

13. La recherche est définie vis-à-vis de l'objet, la ressource en eau potable. L'intérêt des entretiens a ensuite été de constater la difficulté, pour les individus enquêtés, de distinguer, d'une part, la ressource en eau en général, et d'autre part la ressource en eau potable. Le choix a été fait de considérer cette diversité d'énonciation, au niveau des discours fabriqués par les individus enquêtés, comme un critère d'analyse en lui-même, un indicateur participant à l'élaboration de la typologie.

14. La démarche a été développée en étant conscient du biais et de l'influence que cette parole multiple pouvait avoir sur le répondant principal, mais elle lui permettait aussi parfois de mieux réagir et d'apporter sa position personnelle par contraste. 
de dessalement de l'eau de mer n'était pas encore d'actualité ${ }^{15}$.

Les entretiens ont été intégralement retranscrits, puis deux méthodes d'analyse ont été mises en œuvre. D’une part, le classement des réponses par les principaux items dans une grille d'entretien permet la visualisation individuelle des réponses. Les critères d'analyse afin d'orienter les réponses des individus vers une figure principale sont :

- les dimensions de l'environnement auxquelles le discours renvoie : environnement "biophysique », « humain », technique, politique,

- les étendues déployées dans l'espace représenté : étendues proches ou lointaines, confinées ou vastes, précisément décrites ou bien floues, se rapportant à un espace déjà pratiqué ou seulement imaginé,

- les mémoires des épisodes de sécheresse et de pénurie d'eau, articulées avec les mémoires des expériences résidentielles et touristiques personnelles. À ce sujet, il est intéressant de relever les souvenirs des expériences particulières au cours d'un séjour, d'un voyage.

D'autre part, la lecture et relecture linéaire des textes d'entretien a permis de discerner les sens des rapports à l'environnement, physique et social, que le classement par items ne permet pas de faire apparaître. Les deux approches ont donc été complémentaires.

\section{Cing Modes d'habiter L'ESPACE TOURISTIQUE IDENTIFIÉS PAR LA QUESTION DE L'ATTENTION À LA RESSOURCE EN EAU}

Aboutir à une typologie en cinq modes d'habiter paraît intéressant (tableau 1), car cela donne une lisibilité des rapports à l'environnement, mais ce « découpage » est à relativiser pour plusieurs raisons. Il cherche à produire une structure dans et de la société alors qu'existent les dimensions individuelle et plurielle de l'individu. Il procède également, avec une part d'arbitraire, à un classement parce que chaque individu est identifié seulement selon ce qu'il aura dit dans un entretien. Son identité et son rapport à l'environnement peuvent être partiellement autre chose, et il peut finalement relever d'une appartenance à plusieurs modes d'habiter, selon la

15. Le projet d'unité de dessalement à Belle-Île-en-Mer a été d'actualité à partir de l'été 2010. destination touristique interrogée. Chaque individu incorpore et incarne un savoir social (Kaufmann, 2006). Cette incorportation se traduit ici par une figure dominante, mais non exclusive, des modes d'habiter l'espace touristique (tableau 1).

Les avantages de la typologie sont néanmoins de montrer qu'il existe des critères géographiques et sociologiques pertinents de différenciation entre les individus. À partir des items du guide d'entretien, et des discours des individus, les critères de différenciation sont les suivants :

- Le mode d'habiter l'espace de la destination touristique. Certains individus connaissent bien cet espace, d'autres le découvrent. Le mode d'habiter la destination est induit en partie par le style d'habiter les différentes expériences de mobilité touristique. L'origine géographique et sociale des individus (tableau 1) a son importance.

- La représentation de l'espace de la ressource en eau : de la ressource en eau en général, à l'eau potable en particulier, les individus se représentent différents parcours de l'eau, de son origine à son utilisation ${ }^{16}$. Dans cette représentation de la ressource, se place aussi la représentation du risque de pénurie d'eau. Interroger la représentation de cet espace précise alors l'habiter (mode d'habiter in situ, et style d'habiter) de chaque individu.

- La représentation de l'espace des acteurs ne respectant pas (assez) l'eau potable, et des acteurs devant sensibiliser l'ensemble de la population, selon les individus interrogés. C'est ici la représentation de l'espace social et politique qui est questionnée, dans le cadre de la cohabitation des individus en société. Qui doit être sensibilisé, et qui doit prendre la responsabilité de la sensibilisation et de la protection de la ressource?

Le niveau d'expérience du monde conduit à des styles d'habiter, qui sont donc effectifs sur l'ensemble des parcours de vie des individus, et agissent sur le mode d'habiter une destination touristique.

\section{Habiter un environnement " naturel " et technique}

Les individus de ce premier type présentent une sensibilité importante à l'environnement naturel. Ils

16. Le statut d'eau potable est d'ailleurs questionné, le volume d'eau potable étant perçu comme servant à d'autres usages qu'à la consommation humaine. 


\begin{tabular}{|c|c|c|c|c|c|c|c|}
\hline $\mathrm{N}^{\circ}$ & $\begin{array}{c}\text { Lieu de } \\
\text { vacances }\end{array}$ & Hébergement & Domicile & Sexe & Âge & Métier & $\begin{array}{l}\text { Figure principale et non exclusive issue de } \\
\text { l'entretien }\end{array}$ \\
\hline 1 & La Trinité & $\begin{array}{l}\text { Résidence } \\
\text { secondaire }\end{array}$ & Périgord & $\mathrm{H}$ & $60-70$ & Exploitant forestier & Habiter un monde globalisé \\
\hline 2 & Carnac & Camping & Ille-et-Vilaine & $\mathrm{H}$ & $18-30$ & $\begin{array}{l}\text { Technicien environ- } \\
\text { nement }\end{array}$ & $\begin{array}{l}\text { Habiter un environnement naturel et } \\
\text { technique }\end{array}$ \\
\hline 3 & Carnac & Camping & Morbihan & $\mathrm{H}$ & $30-40$ & Militaire & Habiter les lieux médiatiques \\
\hline 4 & La Trinité & Camping & Ille-et-Vilaine & $\mathrm{H}$ & $30-40$ & Chauffeur & Habiter les lieux médiatiques \\
\hline 5 & La Trinité & Camping & Ille-et-Vilaine & F & $60-70$ & Retraitée & Habiter le territoire touristique \\
\hline 6 & Carnac & Camping & Val-de-Marne & $\mathrm{F}$ & $30-40$ & Comptable & Habiter un monde globalisé \\
\hline 7 & La Trinité & Camping & Loire & $\mathrm{H}$ & $30-40$ & Intérimaire & $\begin{array}{l}\text { Habiter un environnement naturel et } \\
\text { technique }\end{array}$ \\
\hline 8 & La Trinité & Camping & Ille-et-Vilaine & $\mathrm{F}$ & $30-40$ & Documentaliste & $\begin{array}{l}\text { Habiter un environnement naturel et } \\
\text { technique }\end{array}$ \\
\hline 9 & La Trinité & Camping & Bas-Rhin & $\mathrm{F}$ & $18-30$ & Employée de banque & $\begin{array}{l}\text { Continuer à habiter le territoire du } \\
\text { quotidien }\end{array}$ \\
\hline 10 & Carnac & Camping & Ille-et-Vilaine & $\mathrm{H}$ & $60-70$ & Retraitée & Habiter le territoire touristique \\
\hline 11 & La Trinité & Camping & Bas-Rhin & $\mathrm{H}$ & $60-70$ & Ingénieur & $\begin{array}{l}\text { Habiter un environnement naturel et } \\
\text { technique }\end{array}$ \\
\hline 12 & La Trinité & Camping & Seine-Maritime & $\mathrm{F}$ & $30-40$ & Secrétaire & $\begin{array}{l}\text { Continuer à habiter le territoire du } \\
\text { quotidien }\end{array}$ \\
\hline 13 & Carnac & Camping & Indre-et-Loire & F & $60-70$ & Retraitée & $\begin{array}{l}\text { Habiter un environnement naturel et } \\
\text { technique }\end{array}$ \\
\hline 14 & Belle-Île & Camping & Calvados & F & $40-50$ & $\begin{array}{l}\text { Employée d'assu- } \\
\text { rances }\end{array}$ & Habiter les lieux médiatiques \\
\hline 15 & Belle-Île & Camping & Seine-Saint-Denis & $\mathrm{H}$ & $40-50$ & Ingénieur du son & Habiter les lieux médiatiques \\
\hline 16 & Belle-île & Camping & Loire & $\mathrm{H}$ & $30-40$ & Éducateur & Habiter les lieux médiatiques \\
\hline 17 & Belle-Île & Camping & Yvelines & $\mathrm{F}$ & $18-30$ & Etudiante & Habiter les lieux médiatiques \\
\hline 18 & Belle-Île & Camping & Loire-Atlantique & F & $40-50$ & Enseignante & Habiter les lieux médiatiques \\
\hline 19 & Belle-Île & Camping & Yvelines & $\mathrm{H}$ & $18-30$ & $\begin{array}{l}\text { Éducateur en envi- } \\
\text { ronnement }\end{array}$ & $\begin{array}{l}\text { Habiter un environnement naturel et } \\
\text { technique }\end{array}$ \\
\hline 20 & Belle-Île & Camping & Alsace & $\mathrm{H}$ & $30-40$ & $\begin{array}{l}\text { Technicien en traite- } \\
\text { ment des eaux }\end{array}$ & Habiter le territoire touristique \\
\hline 21 & Belle-Île & Camping & Côtes d'Armor & $\mathrm{H}$ & $18-30$ & Étudiant & Habiter les lieux médiatiques \\
\hline 22 & Belle-Île & Camping & Hérault & $\mathrm{H}$ & $50-60$ & Enseignant & $\begin{array}{l}\text { Habiter un environnement naturel et } \\
\text { technique }\end{array}$ \\
\hline 23 & Belle-Île & Camping & Calvados & $\mathrm{H}$ & $18-30$ & Élève & $\begin{array}{l}\text { Continuer à habiter le territoire du } \\
\text { quotidien }\end{array}$ \\
\hline 24 & Belle-Île & Camping & Loire-Atlantique & $\mathrm{F}$ & $18-30$ & Etudiante & $\begin{array}{l}\text { Continuer à habiter le territoire du } \\
\text { quotidien }\end{array}$ \\
\hline 25 & Belle-Île & Camping & Indre-et-Loire & $\mathrm{H}$ & $40-50$ & Ingénieur & Habiter les lieux médiatiques \\
\hline 26 & Belle-Île & Camping & Essonne & $\mathrm{H}$ & $40-50$ & $\begin{array}{l}\text { Chef de projets } \\
\text { internationaux }\end{array}$ & $\begin{array}{l}\text { Habiter un environnement naturel et } \\
\text { technique }\end{array}$ \\
\hline 27 & Belle-Île & Camping & Seine-Saint-Denis & $\mathrm{F}$ & $50-60$ & Technicienne & $\begin{array}{l}\text { Habiter un environnement naturel et } \\
\text { technique }\end{array}$ \\
\hline 28 & Belle-Île & Camping & Haute-Savoie & $\mathrm{F}$ & $60-70$ & Retraitée & Habiter le territoire touristique \\
\hline 29 & Belle-Île & Camping & Essonne & $\mathrm{H}$ & $60-70$ & $\begin{array}{l}\text { Retraité directeur de } \\
\text { bureau d'études }\end{array}$ & Habiter le territoire touristique \\
\hline 30 & La Trinité & $\begin{array}{l}\text { Résidence } \\
\text { secondaire }\end{array}$ & Hauts-de-Seine & $\mathrm{F}$ & $50-60$ & Employée & $\begin{array}{l}\text { Habiter un environnement naturel et } \\
\text { technique }\end{array}$ \\
\hline 31 & La Trinité & $\begin{array}{l}\text { Résidence } \\
\text { secondaire }\end{array}$ & Gard & $\mathrm{F}$ & $60-70$ & Retraitée & $\begin{array}{l}\text { Habiter un environnement naturel et } \\
\text { technique }\end{array}$ \\
\hline 32 & La Trinité & $\begin{array}{l}\text { Résidence } \\
\text { d'amis }\end{array}$ & Paris & F & $20-30$ & Hôtesse de l'air & Habiter les lieux médiatiques \\
\hline 33 & La Trinité & $\begin{array}{l}\text { Résidence } \\
\text { secondaire }\end{array}$ & Paris & $\mathrm{H}$ & $70-80$ & Retraité médecin & Habiter les lieux médiatiques \\
\hline
\end{tabular}


Xavier MiCHEL - NOROIS n ${ }^{\circ} 231(2014 / 2)$ p. 45-60

\begin{tabular}{|c|c|c|c|c|c|c|c|}
\hline 34 & La Trinité & $\begin{array}{l}\text { Résidence } \\
\text { secondaire }\end{array}$ & Hauts-de-Seine & $\mathrm{H}$ & $60-70$ & $\begin{array}{l}\text { Ingénieur pharma- } \\
\text { ceutique }\end{array}$ & Habiter le territoire touristique \\
\hline 35 & La Trinité & $\begin{array}{l}\text { Résidence } \\
\text { secondaire }\end{array}$ & Ille-et-Vilaine & $\mathrm{F}$ & $60-70$ & Retraitée & $\begin{array}{l}\text { Habiter un environnement naturel et } \\
\text { technique }\end{array}$ \\
\hline 36 & La Trinité & $\begin{array}{l}\text { Résidence } \\
\text { secondaire }\end{array}$ & Puy-de-Dôme & $\mathrm{F}$ & $60-70$ & Retraitée & Habiter les lieux médiatiques \\
\hline 37 & La Trinité & $\begin{array}{l}\text { Résidence } \\
\text { secondaire }\end{array}$ & Loire-Atlantique & $\mathrm{F}$ & $40-50$ & Kinésithérapeute & $\begin{array}{l}\text { Continuer à habiter le territoire du } \\
\text { quotidien }\end{array}$ \\
\hline 38 & La Trinité & $\begin{array}{l}\text { Résidence } \\
\text { secondaire }\end{array}$ & Yvelines & $\mathrm{F}$ & $50-60$ & Sans emploi & Habiter le territoire touristique \\
\hline 39 & Carnac & Camping & Loire-Atlantique & $\mathrm{H}$ & $40-50$ & Ingénieur bâtiment & $\begin{array}{l}\text { Habiter un environnement naturel et } \\
\text { technique }\end{array}$ \\
\hline 40 & La Trinité & $\begin{array}{l}\text { Résidence } \\
\text { secondaire }\end{array}$ & Ille-et-Vilaine & $\mathrm{H}$ & $70-80$ & Retraité chercheur & $\begin{array}{l}\text { Habiter un environnement naturel et } \\
\text { technique }\end{array}$ \\
\hline 41 & La Trinité & $\begin{array}{l}\text { Résidence } \\
\text { secondaire }\end{array}$ & Loire-Atlantique & $\mathrm{F}$ & $50-60$ & Enseignante & Habiter un monde globalisé \\
\hline 42 & La Trinité & $\begin{array}{l}\text { Résidence } \\
\text { secondaire }\end{array}$ & Paris & $\mathrm{H}$ & $20-30$ & Étudiant & Habiter les lieux médiatiques \\
\hline 43 & La Trinité & $\begin{array}{l}\text { Maison de } \\
\text { location }\end{array}$ & Lille & $\mathrm{H}$ & $30-40$ & $\begin{array}{l}\text { Chef de projets } \\
\text { communication }\end{array}$ & $\begin{array}{l}\text { Continuer à habiter le territoire du } \\
\text { quotidien }\end{array}$ \\
\hline 44 & Belle-Île & Camping & Manche & $\mathrm{H}$ & $20-30$ & Moniteur auto-école & $\begin{array}{l}\text { Habiter un environnement naturel et } \\
\text { technique }\end{array}$ \\
\hline 45 & Belle-Île & Camping & Loire-Atlantique & $\mathrm{F}$ & $40-50$ & Enseignante & $\begin{array}{l}\text { Continuer à habiter le territoire du } \\
\text { quotidien }\end{array}$ \\
\hline 46 & Belle-Île & Camping & Rhône & $\mathrm{F}$ & $30-40$ & Enseignante & Habiter les lieux médiatiques \\
\hline 47 & Belle-île & Camping & Orne & $\mathrm{F}$ & $50-60$ & $\begin{array}{l}\text { Responsable d'ani- } \\
\text { mation }\end{array}$ & Habiter un monde globalisé \\
\hline 48 & Belle-Île & Camping & Somme & $\mathrm{H}$ & $40-50$ & Plombier & $\begin{array}{l}\text { Habiter un environnement naturel et } \\
\text { technique }\end{array}$ \\
\hline 49 & Belle-île & Camping & Ardèche & $\mathrm{F}$ & $40-50$ & Secrétaire & Habiter le territoire touristique \\
\hline 50 & Belle-Île & Camping & Paris & $\mathrm{H}$ & $40-50$ & Enseignant & $\begin{array}{l}\text { Habiter un environnement naturel et } \\
\text { technique }\end{array}$ \\
\hline 51 & Belle-Île & Camping & Puy-de-Dôme & $\mathrm{F}$ & $50-60$ & Attachée territoriale & $\begin{array}{l}\text { Continuer à habiter le territoire du } \\
\text { quotidien }\end{array}$ \\
\hline 52 & Belle-Île & Camping & Paris & $\mathrm{H}$ & $70-80$ & Retraité & $\begin{array}{l}\text { Continuer à habiter le territoire du } \\
\text { quotidien }\end{array}$ \\
\hline
\end{tabular}

Tableau 1 : Caractéristiques des individus interrogés

Interviewees'characteristics

mettent en question la durabilité de la ressource en eau avec un risque de pénurie qu'ils identifient à la fois du fait d'un aléa naturel (la sécheresse) et d'une vulnérabilité sociale. Cet aléa temporaire est représenté par eux dans la destination touristique d'un milieu océanique. Ils développent un rapport étroit, au sens de proche et attentionné, à l'environnement. Dans leurs représentations, ils convoquent des éléments de la nature proches d'eux, et exprimant leur attrait pour les eaux propres, belles et mouvantes ${ }^{17}$. Mais leur mode d'habiter, du côté de leur relation à l'autre en société, est marqué par leur position

17. En restant dans la problématique des rapports à l'eau potable, il est important d'inclure cette représentation plus large de la ressource en eau, pour la définition même de cet « habiter l'environnement "naturel" et technique». en faveur de mesures de restriction et d'interdiction d'usages de l'eau. C'est aux pouvoirs publics selon eux d'organiser ces mesures.

Leur rapport à la ressource en eau est fondé sur une représentation d'un milieu biophysique à la fois de surface et souterrain. Les connaissances de ces individus sur la ressource en eau induisent une représentation d'un espace «vertical », du ciel au sous-sol en passant par la surface de la terre, espace à raisonner en prenant en compte les réserves et leurs limites. En termes de style d'habiter, ce qui les définit est une mobilité importante, touristique et autre. "Une pénurie d'eau, c'est avant tout une diminution importante [...] des nappes phréatiques, donc la pluviométrie, le flux des rivières, donc la diminution 
de la nappe phréatique qui fait qu'on doit prendre des arrêtés préfectoraux pour dire on arrête d'arroser le jardin, on arrête de laver la voiture, on diminue la consommation à la maison, on fait attention, ça, c'est une pénurie d'eau. » (n²6, Belle-Île)

Il s'agit souvent d'individus qui occupent un emploi d'ingénieur ou de technicien, pas forcément lié à l'eau, mais les amenant à mieux connaître la question de la ressource en eau. Leur position n'est cependant pas omnisciente, et ils développent dans leur habitat touristique des démarches de découverte de l'environnement : "c'est aller au CPIE [Centre Permanent d'Initiation à l'Environnement] de Le Palais pour avoir des renseignements sur où estce qu'on ne doit pas aller, est-ce qu'il y a des pratiques à éviter, ici, quand on est comme nous en train de marcher autour de l'île, c'est, après, se renseigner aussi auprès des habitants, c'est pour ça aussi que le fait d'être dans un camping, à la base, une source de collecte d'informations, en rencontrant éventuellement des personnes qui pourraient nous aider, nous faciliter certaines choses. " $\left(\mathrm{n}^{\circ} 22\right.$, Belle-Île $)$

L'expérience est relatée à la fois en tant qu'épisode vécu personnellement, et épisode collectif. L'eau est décrite dans son réseau d'alimentation. Ces individus développent au sujet de l'eau un rapport davantage défini par un fluide intégré à un système technique que par un élément naturel prenant place dans le paysage. "L'année de la canicule [...] je me rappelle plus exactement, mais bon, je pense, entre dix heures, midi, et douze heures, quatre heures, des trucs comme ça, ils faisaient des coupures, on pouvait pas aller prendre de douches. Par contre, les w.-c., et tout, étaient coupés. Il y avait pas le droit d'aller dans les w.-c., c'était la panique [...]. Un camp comme ici, ça doit consommer quelque chose comme eau, parce que là, il y a le robinet qui coule toute la journée, les douches toute la journée, la vaisselle toute la journée. » ( ${ }^{\circ} 11$, La Trinité)

Les moyens de sensibilisation à la préservation de l'eau sont énoncés par rapport à un système social et politique d'encadrement. L'individu soucieux ne se pose pas ici directement en intervenant auprès des personnes proches, autour de lui, mais convoque, dans sa représentation, les acteurs publics pour porter attention à la bonne gestion du réseau d'alimentation (faire en sorte d'éviter les fuites), et amener l'ensemble de la population à des pratiques économes :
"Pour vous, quels moyens seraient les plus efficaces pour faire de la sensibilisation? - Pour moi, je sais même pas si la sensibilisation marcherait. Moi je pense qu'il faut être radical... - [autre personne :] Taper dans le portefeuille des gens. - Non, même pas, il faut, non, pas ça, tu peux pas taper dans le portefeuille des gens, alors que les gens, s'ils ont pas de sous, ils ont pas d'eau, donc ils crèvent. Non, il faut restrictionner [sic] l'eau. " (n², La Trinité)

L'extrait présente ci-dessus le souci à la fois pour l'eau et pour la condition d'autrui, avec la perspective d'un encadrement des usages de l'eau en société. Leurs parcours de mobilité, touristique mais aussi professionnel, amènent ces individus à relativiser l'enjeu de la ressource en eau dans le Morbihan, du fait de leurs pratiques touristiques notamment dans le Sud de la France et le bassin méditerranéen : «Si vous voulez, le vrai sentiment de pénurie, c'était probablement davantage au Liban qu'au Sahara, parce qu'au Sahara [...] c'est une parenthèse, c'est trop différent, on peut pas intégrer qu'on est sur une même planète, dans les mêmes endroits. On fait un voyage, on traverse, de toute façon, on peut pas prendre de douche, puisque..., donc on vit différemment pendant un temps extrêmement court, on se lave pas, de toute façon, et comme il fait très chaud, on transpire pas. " (n³0, La Trinité)

Ces individus allient donc un capital spatial important avec un niveau d'éducation et de connaissances physiques et techniques sur l'eau qui est assez élevé par rapport à l'ensemble de la population, pour poser la question de la préservation de l'eau par un encadrement politique et gestionnaire.

\section{Habiter le territoire touristique}

Ce mode d'habiter est le fait d'individus surtout en résidence secondaire, ou alors en hébergement de plein air depuis de nombreuses années dans la même destination, et qui ont ainsi un certain âge (plus de 50 ans). La connaissance, l'attachement et l'engagement dans et avec cet espace sont développés. Ils ont moins « d'expertise » technique, et activent une relation à l'environnement davantage marquée par la proximité. Habiter implique une cohabitation importante de la population présente, à commencer par l'entourage proche (membres de la famille, voi- 
sins) : l'attention à l'environnement et à la ressource n'est pas reportée sur des responsables publics, mais prise en charge par soi-même. Habiter signifie, pour soi et pour l'autre proche, être responsable, et responsabiliser. La conscience d'habiter le monde est aussi bien développée chez eux, du fait d'expériences touristiques assez nombreuses ailleurs, entre autres dans des pays « chauds». Leurs pratiques passées de voyages et séjours à l'étranger sont importantes. La vulnérabilité et la durabilité sont surtout conçues dans une dimension sociale plus que par rapport à la quantité ou la qualité d'une ressource d'origine naturelle ou du dispositif technique d'alimentation, ou bien alors celui-ci est aperçu à partir des équipements visibles « en surface ». Cet aspect visuel, des phénomènes de surface, des pratiques repérées, distingue sensiblement ces individus habitant le territoire touristique de ceux du type précédent qui sont davantage portés sur l'attention à un système invisible. Ils pratiquent beaucoup ce « territoire » qui est devenu le leur, dans les alentours proches de leur résidence, et au-delà, sur les différents littoraux du sud de la Bretagne : la presqu'île de Quiberon, les dunes d'Etel, le golfe du Morbihan, le littoral du sud Finistère. Leur habiter est davantage fondé sur une sensibilité territoriale et paysagère que « simplement » environnementale, et conduit à porter attention aussi aux différents acteurs du territoire, comme les agriculteurs, et finalement, à l'ensemble d'un territoire. "Quand la période touristique bat son plein, et que l'île est pleine de touristes qui se douchent, plusieurs fois par jour, dans les campings, dans les logements, par rapport aux ressources d'eau de l'île, ce qui est utilisé le reste de l'année, est-ce que ça a une incidence? [...] Toutes ces personnes qui arrivent, de voir les touristes débarquer des bateaux, croiser les gens dans les villes, toutes les maisons, toutes les maisons secondaires qu'on a pu voir dans, pendant les randos ou en balade, et de se dire, quand toutes ces maisons sont remplies et que tout le monde se retrouve présent sur cette île, effectivement, comment ça se passe au niveau de l'eau. » ( ${ }^{\circ}$ 20, Belle-île)

Avec les membres de la famille (enfants) dans l'espace domestique, la position de l'adulte va être ferme mais empreinte de patience et de persuasion : "Je dis aux enfants: sous l'eau, on éteint l'eau, on se lave, et on rince après. Il faut faire attention. " $\left(\mathrm{n}^{\circ} 14\right.$, Belle-Île)

Le contact avec l'autre à l'extérieur peut évoluer vers la remarque, la critique, la réprimande, indui- sant alors une cohabitation difficile, comme avec cet exemple d'expérience du vécu de la sécheresse dans un camping de Belle-Île : "Si la jeunesse de maintenant allait chercher l'eau, elle la gâcherait certainement moins. Voyez, il y a des jeunes, là, qui étaient un peu plus loin. L'année de la pénurie, à Belle-île, lui, je sais qu'il passe une heure sous la douche. [...]. Et je dis: "P., tu sais, cette année, on manque d'eau, il faudra que tu sois... Oh, ben alors là, j'en ai rien à foutre. Moi je prendrais ma douche comme d'habitude." Voilà ce qu'il m'a répondu. Il avait vingt-cinq ans, enfin, vingt ans, j'avais envie de le gifler. Parce que c'est un..., moi j'estime que c'est montrer un égoisme débordant. » ( $n^{\circ} 28$, Belle-Île)

Ce rapport à l'espace étant développé dans la durée, les problèmes de ressource en eau (déficit en quantité et qualité) sont aussi perçus comme ne pouvant être résolus qu'à long terme. La connaissance du territoire permet à ces individus de produire des remarques avisées sur le fonctionnement de dispositifs de gestion de l'eau dans le territoire touristique proche de l'hébergement, à propos de la fermeture puis de la reprise des stations de pompage à Belle-Île :

«Il y a des tennis un peu plus haut, et donc juste à côté, il y a une sorte de vallon, avant, ça m'intriguait, il y avait toujours une petite maison, il y avait que une pompe dedans, et puis il y a à peu près une quinzaine d'années, ils ont tout rasé tout ça, et donc on voyait que c'était la fin du vallon, c'est ce qui alimente, enfin, c'est pas ce qui alimente, ce qui débouche dans l'aber. Et donc, ils avaient supprimé ces petites stations de repompage. C'està-dire que, quand l'eau coulait fortement, elle était renvoyée dans le bassin principal de Belle-Île [...]. Et puis là, depuis quatre, cinq ans, ils ont remis des stations, je trouve que c'est vraiment une bonne idée, de refaire ce qui se faisait avant. Parce que, quand vous allez sur Kerlédan, on voit toujours, il y a des barrages, en pierre, avec une porte, et certainement c'était des barrages d'eau. Donc l'eau était bloquée là, donc le vallon devait réceptionner l'eau, et c'était bloqué avant de repartir dans la mer. Et je pense que, pendant les sécheresses qu'il y a eues, depuis longtemps, sur Belle-île, les gens avaient anticipé de façon à toujours avoir de l'eau. » (n²9, Belle-Île)

Si ce mode d'habiter touristique montre un rapport étroit avec l'environnement local, les positions 
des trois types suivants relèvent, elles, davantage d'une distanciation par rapport à l'environnement de la destination.

\section{Continuer à habiter... le territoire du quotidien}

Les individus concernés développent une référence importante à leur territoire du quotidien. Leur pratique de la destination touristique locale est souvent nouvelle, même s'il s'agit d'habitués de la Bretagne. Pour ces catégories sociales intermédiaires, leurs séjours touristiques, en famille, se situent par ailleurs surtout dans d'autres régions de France plus qu'à l'étranger. Leur méconnaissance de l'environnement de la destination par rapport à une bonne connaissance de leur territoire du quotidien les amène à se reporter au quotidien pour concevoir, par analogie, la question de l'eau et de l'environnement dans la destination touristique. Il n'y a pas d'attachement à la destination ni d'appropriation au moyen d'une résidence régulière. Leur référence à un territoire les conduit aussi à concevoir la voie de la durabilité sur le long terme, à partir de leurs expériences et de leur connaissance de la société locale dans laquelle ils vivent au quotidien, et de laquelle ils font ressortir les bonnes conduites. En termes d'environnement, leur image de la vulnérabilité du territoire repose beaucoup plus sur des paysages desséchés, des exemples d'incendie, que sur une ressource en eau à préserver. Les situations de sécheresse sont même parfois bien vécues et représentées :

"- La sécheresse, c'est pas spécialement négatif. Voire même que ça peut être très positif dans le nord de la France.

- Développez.

- Ça veut dire qu'il fait beau, voilà, ça nous va bien, et on a un petit peu de temps plus agréable...

— [Deuxième répondant :] Ou en Bretagne.

- En Bretagne. Moi je connais moins bien, mais ça devient quelque part plus agréable. » ( $\mathrm{n}^{\circ} 43$, La Trinité)

L'attention est portée aux équipements et pratiques hydro-économes pour justifier que le rapport de la société dans son ensemble à la ressource en eau est meilleur aujourd'hui qu'avant. Le vécu local est ainsi étendu dans une représentation au reste de l'espace. «Je pense que on est équipés pour pallier à ça, oui. Il y a des bassins de rétention qui alimentent les régions. [...] Les gens qui continuent à arroser leurs jardins, c'est des gens qui ont des puits, par exemple, des puits qui tarissent pas l'été, donc eux, ils peuvent se permettre d'arroser encore leur jardin. " (n³7, La Trinité)

\section{Habiter les lieux médiatiques}

Ce mode d'habiter concerne plusieurs catégories de personnes : des jeunes, qui n'ont pas encore développé (ou intériorisé pour pouvoir l'exprimer) beaucoup d'expériences des espaces et donc de rapports à l'environnement, des familles de catégories populaires avec une faible mobilité, peu de déplacements lointains et séjournant en camping, mais aussi des résidents secondaires plus aisés. Le style d'habiter touristique est un assemblage de "sauts de puce » d'un lieu à l'autre, mais sans qu'un système de représentation unifié entre les lieux ne se développe. Leurs réactions vis-à-vis de la ressource en eau les conduisent à évoquer des lieux emblématiques, qui sont cités sans argumentation. Cette dimension médiatique signifie que, en complément de leurs expériences, c'est l'image de lieux véhiculée par les médias qui va importer dans l'élaboration de ce qu'ils exprimeront. Vis-à-vis de ces discours, le chercheur est amené à se questionner sur la validité du matériau collecté : représente-t-il bien le rapport de l'individu à l'environnement? N'y a-t-il pas autre chose que ces évocations de lieux emblématiques et symboliques, mais qui reste inexprimé? Il ressort aussi de leurs discours un rapport particulier à la temporalité naturelle et sociale : le passé est évoqué, notamment avec des épisodes ponctuels marquants de crises environnementales, et l'avenir est projeté à partir de là, mais sans que le présent ne soit vraiment décrit. Leur attention personnelle à la ressource en eau n'est pas bien relatée, la question de sa durabilité n'est pas énoncée, et les acteurs responsables et/ou fautifs ne sont pas non plus identifiés. La difficulté à réagir par rapport à l'examen d'une situation se présente alors :

«-À Belle-île, il y avait eu, parce que si je comprends bien, l'eau arrive du continent.

— [Second répondant :] Non.

- Mais alors pourquoi c'était...? 
- [Second répondant :] Ils en manquaient sur l'île, justement. Donc il fallait en apporter.

- [Enquêteur :] Parce que pour vous, l'eau arrive du continent?

- Moi je suis nulle, je sais rien du tout. Je me souviens, c'était des réserves, des réservoirs qui arrivaient du continent, donc je me dis tiens, il doit y avoir quelque chose. On nous avait dit qu'il y avait plus d'eau.

— [Second répondant :] Non, il y a des sources, si, il y a un château d'eau, on est passé devant tout à l'heure. Mais visiblement il y a déjà eu, oui. » (n 14, Belle-Île)

Seules certaines images façonnent les souvenirs des individus, du fait d'un défaut de système d'explication.

La conscience de la préservation de la ressource par rapport à soi et dans son espace de vie peut alors être difficilement conçue : "J'ai toujours consommé pas mal d'eau, je fais couler beaucoup l'eau, je suis une grosse consommatrice d'eau, et l'aspect problématique, c'est qu'on puisse en manquer. Quand on nous dit : n'arrosez plus votre jardin, ça, ça me pose un problème, ou limiter notre consommation, oui. » (n³6, La Trinité)

En parallèle, l'inquiétude vis-à-vis de l'avenir est plus forte chez ces individus, du fait de ce manque d'explications. Le seul espace concret vraiment représenté est celui qui est entre l'individu et le robinet. Au-delà, viennent assez directement les lieux médiatiques des catastrophes : sécheresse du désert, pollutions, fonte de la banquise, et ce sont les acteurs de la diffusion des images : célébrités télévisées, etc., qui sont perçus comme pouvant jouer un rôle dans la sensibilisation envers les ressources.

\section{Habiter un monde globalisé}

La dimension globale et détachée par rapport aux environnements concrets est aussi développée chez un dernier type d'individus, peu nombreux (quatre) parmi le groupe de 52 individus enquêtés, mais qui formulent une position tout à fait spécifique au sujet de l'évolution de l'environnement et de la ressource en eau. Ils développent un système d'explication globale de l'environnement, auquel ils relient la destination touristique Morbihan (comme d'autres destinations).
« Si ça arrive, on est quand même dans un environnement privilégié, si notre environnement à nous, privilégié, est touché, ça veut dire que, malheureusement, il y aura beaucoup d'autres gens qui auront souffert, même plus que souffert, de ça avant nous. Je parle bien, en étant là où je vis, voilà. Le jour où, vraiment, c'est que, malheureusement, enfin, j'ai peur que, malheureusement, il y ait beaucoup d'autres pays qui aient été touchés encore plus gravement avant nous [...], parce que, tout est mondial, donc s'il y a un problème d'eau, s'il y a un problème de réchauffement climatique, personne n'y échappera. » ( $\mathrm{n}^{\circ}$ 6, La Trinité)

La technologie est ici finalement déconnectée d'un problème représenté seulement par une image de matérialité physique. Le risque conçu chez ces individus est celui de problèmes provenant des Suds et remontant vers les pays du Nord, donc vers l'ouest de la France: "J'espère que non, ne pas connaître ça, et que mes enfants, mes petits-enfants ne le connâेtront pas, mais je vois, par exemple, enfin j'entends des émissions sur ce qui se passe au sud de l'Espagne, qui devient le Sahara qui remonte, la sécheresse au sud de l'Espagne, bon, je me dis que ça pourrait encore remonter et..., j'espère ne pas le connaître, qu'on ne le connaîtra pas. » ( $\mathrm{n}^{\circ} 41$, La Trinité).

Il est difficile pour eux de penser la durabilité de la ressource, car ils sont marqués par la représentation d'effets catastrophiques du changement global sur les territoires, sans que les acteurs territoriaux ne puissent vraiment agir.

\section{Conclusion}

Les modes d'habiter les destinations touristiques sont différenciés selon les parcours de mobilité et de résidences de chacun. Cette influence joue sur la manière dont les individus instaurent leurs relations à l'environnement de la destination, ce qu'ils désignent comme nature, mais aussi comme technologie et comme « les autres », autour d'eux et jusqu'à l'échelle mondiale, en passant par différents agencements pratiques et imaginaires. Les types apparaissent ainsi, en fonction des principaux critères de différenciation identifiés au préalable, et qui se rangent dans le tableau 2.

La caractérisation de ces modes et styles d'habiter renvoie à des spatialités différentes, ce qui peut 


\begin{tabular}{|c|c|c|c|c|c|}
\hline & $\begin{array}{l}\text { Habiter un envi- } \\
\text { ronnement « natu- } \\
\text { rel » et technique }\end{array}$ & $\begin{array}{l}\text { Habiter le terri- } \\
\text { toire touristique }\end{array}$ & $\begin{array}{c}\text { Continuer à habi- } \\
\text { ter le territoire du } \\
\text { quotidien }\end{array}$ & $\begin{array}{l}\text { Habiter les lieux } \\
\text { médiatiques }\end{array}$ & $\begin{array}{l}\text { Habiter un monde } \\
\text { globalisé }\end{array}$ \\
\hline $\begin{array}{l}\text { Mobilités et mode } \\
\text { d'habiter la desti- } \\
\text { nation touristique }\end{array}$ & $\begin{array}{l}\text { Mobilités touris- } \\
\text { tiques importantes, } \\
\text { catégories sociales } \\
\text { élevées ou métiers } \\
\text { liés à l'environne- } \\
\text { ment. }\end{array}$ & $\begin{array}{l}\text { Mobilités touris- } \\
\text { tiques importantes, } \\
\text { séjours réguliers } \\
\text { dans le Morbihan, } \\
\text { catégories sociales } \\
\text { élevées }\end{array}$ & $\begin{array}{l}\text { Mobilités touris- } \\
\text { tiques limitées à la } \\
\text { France, découverte } \\
\text { du Morbihan, } \\
\text { catégorie sociale } \\
\text { intermédiaire }\end{array}$ & $\begin{array}{l}\text { Mobilités touris- } \\
\text { tiques dispersées, } \\
\text { catégories sociales } \\
\text { populaires ou } \\
\text { élevées. }\end{array}$ & $\begin{array}{l}\text { Mobilités tou- } \\
\text { ristiques surtout } \\
\text { limitées à la France, } \\
\text { catégories sociales } \\
\text { variées. }\end{array}$ \\
\hline $\begin{array}{l}\text { Représentation } \\
\text { de l'espace de la } \\
\text { ressource en eau } \\
\text { potable, représen- } \\
\text { tation du risque de } \\
\text { pénurie d'eau }\end{array}$ & $\begin{array}{l}\text { Espace de res- } \\
\text { source aérien, de } \\
\text { surface, souterrain. } \\
\text { Aléa sécheresse } \\
\text { possible temporai- } \\
\text { rement. }\end{array}$ & $\begin{array}{l}\text { Espace de res- } \\
\text { source de surface. } \\
\text { Vulnérabilité } \\
\text { sociale par manque } \\
\text { de préservation. }\end{array}$ & \begin{tabular}{|l|} 
Espace de res- \\
source : réseau \\
technique. \\
Durabilité de la res- \\
source en progrès \\
par les équipements \\
hydro-économes. \\
\end{tabular} & $\begin{array}{l}\text { Espace de res- } \\
\text { source vu surtout } \\
\text { par rapport au passé } \\
\text { et à l'avenir. Le } \\
\text { présent est limité à } \\
\text { l'apport du robinet. }\end{array}$ & $\begin{array}{l}\text { Espace de res- } \\
\text { source représenté } \\
\text { globalement, sans } \\
\text { frontières. Risque } \\
\text { lié au changement } \\
\text { global. }\end{array}$ \\
\hline $\begin{array}{l}\text { Représentation du } \\
\text { rôle de chacun en } \\
\text { société pour pré- } \\
\text { server la ressource } \\
\text { en eau potable }\end{array}$ & $\begin{array}{l}\text { Restriction et } \\
\text { interdiction par les } \\
\text { pouvoirs publics. }\end{array}$ & $\begin{array}{l}\text { Porter attention soi- } \\
\text { même et sensibili- } \\
\text { ser son entourage. }\end{array}$ & $\begin{array}{l}\text { Satisfaction } \\
\text { relative du fait des } \\
\text { pratiques hydro- } \\
\text { économes déjà en } \\
\text { place. }\end{array}$ & $\begin{array}{l}\text { Rôle des médias, } \\
\text { des campagnes } \\
\text { médiatiques pour } \\
\text { sensibiliser. }\end{array}$ & $\begin{array}{l}\text { Rôle des acteurs } \\
\text { minoré dans une } \\
\text { vision catastro- } \\
\text { phiste de risque de } \\
\text { pénurie. }\end{array}$ \\
\hline
\end{tabular}

Tableau 2 : Habiter l'espace touristique et porter attention à la ressource en eau potable Inhabiting tourist space and caring for drinking water resources

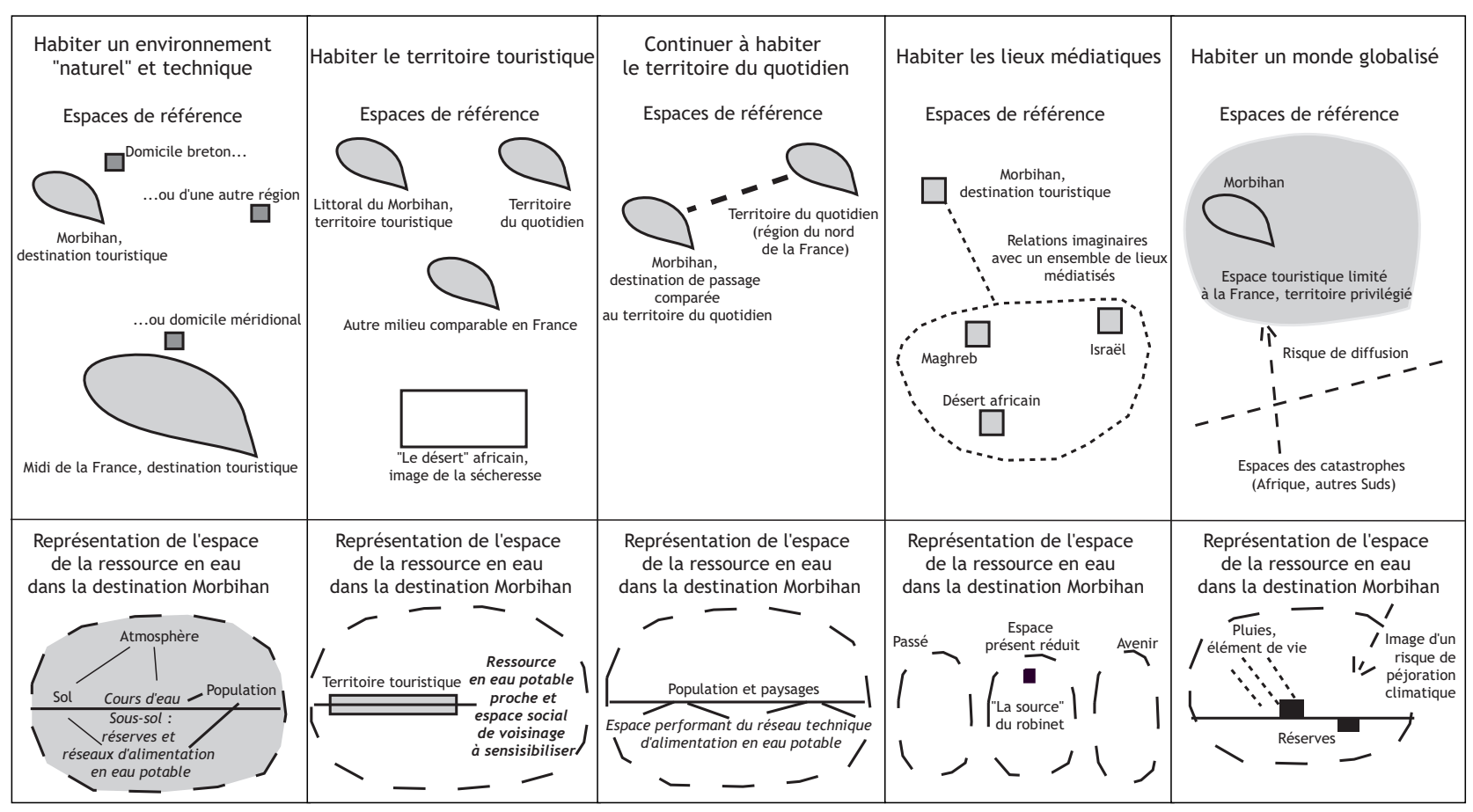

Figure 2 : Espaces de référence et représentations de la ressource en eau potable dans le Morbihan Reference spaces and representations of drinking water resources in Morbihan

être traduit par des représentations graphiques (figure 2). À partir de l'analyse des entretiens, ces représentations forment une interprétation visuelle des espaces de référence des individus. Chaque in- dividu fait ressortir une figure dominante à laquelle il peut être lié, mais ce n'est pas un rattachement exclusif. Par exemple, un individu peut « habiter le territoire touristique » et aussi « habiter un monde 
globalisé ». Habiter un environnement « naturel » et technique renvoie à des savoirs et expériences développées sur l'environnement (figure 2), alors que la figure « habiter les lieux médiatiques » correspond à un rapport à la ressource en eau potable davantage limité à une relation plus réduite avec le seul espace d'alimentation visible : le robinet. Entre ces deux figures opposées, plusieurs représentations de l'espace existent, où les ancrages à des territoires importent beaucoup dans la construction des modes d'habiter et l'attention pour la ressource en eau potable :

- territoire touristique du Morbihan pour ceux qui y ont leurs habitudes et qui y développent un espace de voisinage, jusqu'à porter attention aux usages de l'eau potable par les voisins,

- territoire du quotidien (celui du domicile permanent) pour des touristes de passage dans le Morbihan, qui portent attention à l'eau potable dans la destination touristique comme chez eux,

- territoire français et occidental privilégié, pour des individus se plaçant dans un monde globalisé et qui imaginent ainsi une menace pouvant les affecter. Ils développent une attention à l'eau potable davantage fondée sur sa valeur de bien précieux universel.

Ces différentes positions des touristes renvoient à des représentations de l'alimentation en eau potable diverses et assez décalées par rapport à la réalité du réseau technique. Seuls les individus qui forment la catégorie "Habiter un environnement "naturel" et technique » développent vraiment la représentation du réseau d'alimentation.

Enfin, un espace touristique, en tant que rassemblement de populations différentes par leurs origines, leurs mobilités et leurs territorialités vis-à-vis de la destination « Morbihan ", amène à réfléchir à l'enjeu de la cohabitation à l'intérieur de la société, pour faire comprendre l'importance de la préservation de la ressource en eau potable. À ce sujet, il apparaît des catégories motrices, comme « habiter le territoire touristique », mais aussi : " habiter un environnement naturel et technique », à condition que les individus qui les incarnent appellent à la préservation de la ressource en eau de manière positive envers les autres, pour que les différents modes d'habiter ne se positionnent pas en oppositions.

\section{Remerciements}

L'auteur remercie le ministère de l'Écologie et du Développement Durable et le PUCA pour les moyens accordés afin de permettre la réalisation de cette recherche dans le cadre du programme RDT2.

\section{Bibliographie}

Bachelard G., 1942. L'Eau et les rêves Essai sur l'imagination de la matière, Paris, José Corti, 221 p.

Billaud J.-P., Michel X. (2010) - L'alimentation en eau potable dans le Morbihan : représentations, perceptions et réductions $d u$ risque de pénurie d'eau dans un territoire touristique, UMR LADYSS, Rapport pour le programme Risques, décision, territoire $\mathrm{n}^{\circ} 2$, Ministère de l'écologie, $115 \mathrm{p}$.

Buzinde C., Manuel-Navarette D., Kerstetter D., 2010. Representations and adaptations to climate change, Annals of Tourism Research, vol. 37, n³ 3, p. 581-603.

Chiron T., 2007a. Quel risque de pénurie d'eau sur les îles du Ponant?, Norois, vol. 202, n 1 , p. 73-86.

Chiron T., 2007b. Quelle gestion durable des ressources en eau et du risque de pénurie d'eau sur les petites îles? Application aux iles de Bretagne (France). Thèse de doctorat en Géographie, Université de Bretagne Occidentale, 412 p.

Dolnicar S., Crouch G.I., Long P., 2008. Environment-friendly tourists : what do we really know about them?, Journal of Sustainable Tourism, vol. 16, n² 2, p. 197-210.

Dubreuil V., 1997. La sécheresse dans la France de l'Ouest: une contrainte climatique trop souvent oubliée, Sécheresse, vol. $8, n^{\circ} 1$, p. 47-55.

Emélianoff C., 2013. Environnement, in LÉvy J., Lussault M. (dir.), Dictionnaire de la géographie et de l'espace des sociétés, Paris, Belin, p. 342-343.

Gössling S., 2006. Tourism and Water, in Gössling S., Hall C.M. (dir.), Tourism and Global Environmental Change, Londres/New York, Routledge, p. 180-194.

Gössling S., Peeters P., Hall C.M., Ceron J.-P., Dubois G., Lehmann L.V., Sсотt D., 2012. Tourism and Water Use : Supply, Demand and Security An international review, Tourism Management, vol. 33, $\mathrm{n}^{\circ}$ 1, p. 1-15.

Hellier E., Carré C., Dupont N., Laurent F., Vaucelle S., 2009. La France La ressource en eau Usages, gestions et enjeux territoriaux, Paris, Armand Colin, 309 p.

Hoyaux A.-F., 2003. Les constructions des mondes de l'habitant : éclairage pragmatique et herméneutique, Cybergeo European Journal of Geography, document 232, [http://cybergeo.revues.org/3401].

Kaufmann J.-C., 2006. L'entretien compréhensif, Paris, Armand Colin, $126 \mathrm{p}$.

Maulion H., 2009. Exploration de récits paysagers sur les littoraux de Belle-Île-en-Mer (France) et de la péninsule de Dingle (Irlande), Norois, vol. 213, n 4, p. 41-57.

Michel X., 2005. Associer le quotidien, les vacances et les excursions pour évaluer les espaces de qualité et de bien- 
être des habitants de différents milieux (urbain, périurbain, rural, montagnard), in Fleuret S. (dir.), Espaces, Qualité de vie et Bien-être, Angers, Presses Universitaires d'Angers, p. 277-285.

Miller G., Rathouse K., Scarles C., Holmes K., Tribe J., 2010. Public Understanding of Sustainable Tourism, Annals of Tourism Research, n 3, p. 627-645.

Mounier J., Dubreuil V., Le Hénaff F., 1993. Sécheresse et rivières en Bretagne occidentale, in Griselin M. (dir.), L'eau, la terre et les hommes Hommage à René Frécaut, Nancy, Presses Universitaires de Nancy, p. 263-272.

Ollivro J., 2005. Les classes mobiles, L'Information géographique, vol. 60, $\mathrm{n}^{\circ} 3$, p. 28-44.

Potier F., 2002. La République du temps libre n'est pas une et indivisible, in VIARD J. (dir.), La France des temps libres et des vacances, La Tour d'Aigues, Éditions de l'Aube/Datar, p. $149-163$.

Raymond R., 2004. De quelle nature parle-t-on ?, Strates, $n^{\circ} 11$, p. 43-56.

Sтоск M., 2005. Les sociétés à individus mobiles : vers un nouveau mode d'habiter?, EspacesTemps.net, Textuel, 25.05.2005, [http://espacestemps.net/document1353.html].
Sтоск M., 2006. L'hypothèse de l'habiter polytopique, EspacesTemps.net, Textuel, 26.02.2006, [http:/lespacestemps.net/ document 1853.html].

Sтоск M., 2012. «Faire avec de l'espace » : pour une approche de l'habiter par les pratiques, in Frelat-Kahn B., Lazzaвотті O. (dir.), Habiter Vers un nowveau concept, Paris, Armand Colin, p. 57-74.

Terrier C. (dir.) 2006. Mobilité touristique et population présente. Les bases de l'économie présentielle des départements, Paris, Direction du Tourisme, 128 p.

URry J., 2005. Sociologie des mobilités Une nouvelle frontière pour la sociologie?, Paris, Armand Colin, 253 p.

Vacher L., Vye D., 2012. Penser l'habiter à travers la pratique des lieux touristiques par les excursionnistes, touristes et résidents, in Frelat-Kahn B., Lazzarotti O. (dir.), Habiter Vers un nouveau concept, Paris, Armand Colin, p. 197-210.

Viard J., 1984. Penser les vacances, Le Paradou, Actes Sud, 204 p. 